\title{
On the Solution of Skew-Symmetric Shifted Linear Systems
}

\author{
T. Politi ${ }^{1}$ and A. Pugliese ${ }^{2}$ \\ 1 Dipartimento di Matematica, Politecnico di Bari, \\ Via Amendola 126/B, I-70126 Bari (Italy) \\ politi@poliba.it \\ 2 School of Mathematics, Georgia Institute of Technology, \\ Atlanta, GA 30332 U.S.A. \\ pugliese@math.gatech.edu
}

\begin{abstract}
In this paper we consider the problem of solving a sequence of linear systems with coefficient matrix $A_{\alpha}=I+\alpha A$ (or $A_{\alpha}=\alpha I+A$ ), where $\alpha$ is a real paramater and $A$ is skew-symmetric matrix. We propose to solve this problem exploiting the structure of the Schur decomposition of the skew-symmetric matrix and computing the Singular Value Decomposition of a bidiagonal matrix of halved size.
\end{abstract}

\section{Introduction}

In this paper we consider the solution of a sequence of linear systems of the form

$$
A_{\alpha} \boldsymbol{x}_{\alpha}=\boldsymbol{b}_{\alpha}
$$

where

$$
A_{\alpha}=I+\alpha A
$$

or

$$
A_{\alpha}=\alpha I+A
$$

with $I$ identity matrix of order $n, \alpha$ positive real parameter belonging to $] 0, \alpha_{\max }$ ] and $A$ is a real skew-symmetric matrix of order $n$. The question is how to solve efficiently the linear systems for subsequent values of the shift $\alpha$. The goal is to obtain a solution procedure that is cheaper, in terms of total solution costs, trying to save much computations as possible. In [2,3] the authors consider the solution of a sequence of linear systems (12)-(2) having $A$ symmetric and positive definite. In this case they try to obtain an efficient preconditioning matrix to save computational operations when the parameter $\alpha$ changes, observing that using the same preconditioner for different values of $\alpha$ brings to a very slow convergence. Sequences of linear systems of the form (1)-(2) arise in different fields of applied mathematics. If we consider the numerical solution of a Kortwegde Vries partial differential equation using a particular discretization (see [4]) a differential system

$$
y^{\prime}(t)=A(y) y(t)
$$


with initial condition $y(0)=y_{0}$ has to be solved. Applying numerical schemes of the integration, at each step, a sequence of linear systems (11)-(2) has to be solved.

The paper is organized as follows: in Section 2 we derive the structure for a decomposition of matrix (2), in Section 3 we analyze the computational cost of the proposed method, while in Section 4 possible applications are described. The following theorem is a general result that will be useful later.

Theorem 1. Let $A$ be the following $n \times n, n$ even, square matrix

$$
A=\left[\begin{array}{cc}
B & C \\
-C & B
\end{array}\right]
$$

with $C, B \in \mathbb{R}^{m \times m}$ symmetric matrices and $m=n / 2$. If $A$ is nonsingular then $A^{-1}$ retains the same structure of $A$, i.e.

$$
A^{-1}=\left[\begin{array}{cc}
X & Y \\
-Y & X
\end{array}\right]
$$

with $X, Y \in \mathbb{R}^{m \times m}$ symmetric matrices.

\section{The Decomposition of the Matrix $I+\alpha A$}

The factorization technique that we describe in this section has the same of starting point of the one shown in 5 in order to compute the exponential of the skew-symmetric matrix $A$ times a unitary norm vector $\boldsymbol{v}$, and, in general, to compute analytic matrix functions. First of all we consider the problem to find the Schur decomposition of the skew-symmetric matrix $A$. Hence we consider the real skew symmetric matrix $A \in \mathbb{R}^{n \times n}$, a vector $\boldsymbol{q}_{1} \in \mathbb{R}^{n}$, such that $\left\|\boldsymbol{q}_{1}\right\|_{2}=1$, the Krylov matrix $K\left(A, \boldsymbol{q}_{1}, m\right)=\left[\begin{array}{lll}\boldsymbol{q}_{1} & A \boldsymbol{q}_{1} & A^{2} \boldsymbol{q}_{1} \ldots A^{m-1} \boldsymbol{q}_{1}\end{array}\right] \in \mathbb{R}^{n \times m}$ and the Krylov subspace $\mathcal{K}_{m}=\operatorname{span}\left\{\boldsymbol{q}_{1}, A \boldsymbol{q}_{1}, \ldots, A^{m-1} \boldsymbol{q}_{1}\right\}$. The following result states the conditions for the existence of a Hessenberg form of $A$ (see [6]).

Theorem 2. Suppose $Q=\left[\begin{array}{lll}\boldsymbol{q}_{1} & \boldsymbol{q}_{2} \ldots \boldsymbol{q}_{n}\end{array}\right] \in \mathbb{R}^{n \times n}$ is an orthogonal matrix. Then $Q^{\top} A Q=H$ is an unreduced Hessenberg matrix if and only if $R=Q^{\top} K\left(A, \boldsymbol{q}_{1}, n\right)$ is nonsingular and upper triangular.

Thus, when $K\left(A, \boldsymbol{q}_{1}, n\right)$ is of full rank $n$, from the QR factorization of $K\left(A, \boldsymbol{q}_{1}, n\right)$, it follows that an unreduced Hessenberg form $H$ of $A$ exists. The Hessenberg decomposition, $A=Q H Q^{\top}$, is essentially unique when the first column $\boldsymbol{q}_{1}$ of $Q$ is fixed and its unreduced Hessenberg form $H$ is skew-symmetric and possesses the following tridiagonal structure

$$
H=\left[\begin{array}{ccccc}
0 & -h_{2} & 0 & \ldots & 0 \\
h_{2} & 0 & -h_{3} & \ddots & \vdots \\
0 & \ddots & \ddots & \ddots & 0 \\
\vdots & \ddots & h_{n-1} & 0 & -h_{n} \\
0 & \ldots & 0 & h_{n} & 0
\end{array}\right]
$$


For skew-symmetric matrices the reduction to the above tridiagonal form can be performed using the following Lanczos tridiagonalization process:

Let $\boldsymbol{q}_{1}$ be a vector of $\mathbb{R}^{n}$ with $\left\|\boldsymbol{q}_{1}\right\|=1$ and set $h_{1}=0$ and $\boldsymbol{q}_{0}=0$.

$$
\begin{aligned}
& \text { for } j=1: n \\
& \qquad \begin{array}{l}
\boldsymbol{w}_{j}=A \boldsymbol{q}_{j}+h_{j} \boldsymbol{q}_{j-1} \\
h_{j+1}=\left\|\boldsymbol{w}_{j}\right\| \\
\boldsymbol{q}_{j+1}=\boldsymbol{w}_{j} / h_{j+1}
\end{array} \\
& \text { end }
\end{aligned}
$$

We have to notice that, in exact arithmetic, the above algorithm is equivalent to the Arnoldi process applied to skew symmetric matrices. It provides an orthogonal matrix $Q=\left[\begin{array}{llll}\boldsymbol{q}_{1} & \boldsymbol{q}_{2} & \ldots & \boldsymbol{q}_{n}\end{array}\right] \in \mathbb{R}^{n \times n}$ such that $Q^{\top} A Q=H$ where $H$ is the tridiagonal matrix (3). Moreover, it allows one to take full advantage of the possible sparsity of $A$ due to the matrix-vector products involved. However, in floating-point arithmetic the vectors $\boldsymbol{q}_{j}$ could progressively lose their orthogonality, in this case, the application of a re-orthogonalization procedure is required 6, 7]. We suppose $n$ to be even, but the case of $n$ odd may be approached in a similar way. Let us consider the permutation matrix

$$
P=\left[\boldsymbol{e}_{1}, \boldsymbol{e}_{3}, \ldots, \boldsymbol{e}_{n-1}, \boldsymbol{e}_{2}, \boldsymbol{e}_{4}, \ldots, \boldsymbol{e}_{n}\right],
$$

where $\boldsymbol{e}_{i}$ is the $i$-th vector of the canonical basis of $\mathbb{R}^{n}$. Then, if $H$ is as in (3) we have

$$
P^{\top} H P=\left[\begin{array}{cc}
0 & -B \\
B^{\top} & 0
\end{array}\right]
$$

where $B$ is the lower bidiagonal square matrix of size $m=n / 2$ given by:

$$
B=\left[\begin{array}{ccccc}
h_{2} & 0 & \ldots & \ldots & 0 \\
-h_{3} & h_{4} & \ldots & & 0 \\
0 & -h_{5} & \ddots & \ddots & \vdots \\
\vdots & \ddots & \ddots & h_{n-2} & 0 \\
0 & \ldots & 0 & -h_{n-1} & h_{n}
\end{array}\right]
$$

Since all diagonal and subdiagonal entries of $B$ are non zero, the $m$ singular values of $B$ are distinct and different from zero.

Let us consider the singular value decomposition of $B$

$$
B=U \Sigma V^{\top},
$$

with $\Sigma=\operatorname{diag}\left(\sigma_{1}, \sigma_{2}, \ldots, \sigma_{m}\right)$ and $\sigma_{1}>\sigma_{2}>\ldots \sigma_{m}>0$, and the orthogonal $n \times n$ matrix

$$
W=\left[\begin{array}{cc}
U & 0 \\
0 & V
\end{array}\right]
$$

Hence

$$
W^{\top} P^{\top} H P W=\left[\begin{array}{cc}
U^{\top} & 0 \\
0 & V^{\top}
\end{array}\right]\left[\begin{array}{cc}
0 & -B \\
B^{\top} & 0
\end{array}\right]\left[\begin{array}{cc}
U & 0 \\
0 & V
\end{array}\right]=\left[\begin{array}{cc}
0 & -\Sigma \\
\Sigma & 0
\end{array}\right] .
$$


The matrix $I+\alpha A$ can be decomposed as

$$
I+\alpha A=Q P W(I+\alpha \widehat{\Sigma}) W^{\top} P^{\top} Q^{\top}
$$

where

$$
\widehat{\Sigma}=\left[\begin{array}{cc}
O & -\Sigma \\
\Sigma & O
\end{array}\right]
$$

If we have to solve the sequence of linear systems (12)-(2) we could exploit the factorization (6) computing vector $\boldsymbol{x}_{\alpha}$, for a given value $\alpha$ through the following steps:

1. Compute $\boldsymbol{y}_{\alpha}=Q^{\top} \boldsymbol{b}_{\alpha}$;

2. Compute $\boldsymbol{c}_{\alpha}=W^{\top} P \boldsymbol{y}_{\alpha}$;

3. Solve the linear system

$$
(I+\alpha \widehat{\Sigma}) \boldsymbol{u}_{\alpha}=\boldsymbol{c}_{\alpha} \quad \Leftrightarrow \quad\left[\begin{array}{cc}
I_{m} & -\alpha \Sigma \\
\alpha \Sigma & I_{m}
\end{array}\right] \boldsymbol{u}_{\alpha}=\boldsymbol{c}_{\alpha}
$$

4. Compute $\boldsymbol{x}_{\alpha}=Q P W \boldsymbol{u}_{\alpha}$.

The solution of (7) is very simple, in fact exploiting Theorem 1 it is easy to compute the inverse of the matrix $I+\alpha \widehat{\Sigma}$. In fact:

$$
\left[\begin{array}{cc}
I_{m} & -\alpha \Sigma \\
\alpha \Sigma & I_{m}
\end{array}\right]^{-1}=\left[\begin{array}{cc}
X & Y \\
-Y & X
\end{array}\right]
$$

where

$$
\begin{aligned}
& X=\left(I_{m}+\alpha^{2} \Sigma^{2}\right)^{-1} \\
& Y=\alpha \Sigma\left(I_{m}+\alpha^{2} \Sigma^{2}\right)^{-1}
\end{aligned}
$$

and, explicitly

$$
\begin{aligned}
& X=\operatorname{diag}\left(\left(1+\alpha \sigma_{1}^{2}\right)^{-1},\left(1+\alpha \sigma_{2}^{2}\right)^{-1}, \ldots,\left(1+\alpha \sigma_{m}^{2}\right)^{-1}\right) \\
& Y=\operatorname{diag}\left(\alpha \sigma_{1}\left(1+\alpha \sigma_{1}^{2}\right)^{-1}, \alpha \sigma_{2}\left(1+\alpha \sigma_{2}^{2}\right)^{-1}, \ldots, \alpha \sigma_{m}\left(1+\alpha \sigma_{m}^{2}\right)^{-1}\right) .
\end{aligned}
$$

Decomposing the vectors $\boldsymbol{u}_{\alpha}$ and $\boldsymbol{c}_{\alpha}$ as

$$
\boldsymbol{u}_{\alpha}=\left[\begin{array}{l}
\boldsymbol{u}_{1} \\
\boldsymbol{u}_{2}
\end{array}\right], \quad \boldsymbol{c}_{\alpha}=\left[\begin{array}{l}
\boldsymbol{c}_{1} \\
\boldsymbol{c}_{2}
\end{array}\right]
$$

with $\boldsymbol{u}_{1}, \boldsymbol{u}_{2}, \boldsymbol{c}_{1}, \boldsymbol{c}_{2} \in \mathbb{R}^{m}$, the solution of system (7) is

$$
\begin{aligned}
& \boldsymbol{u}_{1}=\left(I_{m}+\alpha^{2} \Sigma^{2}\right)^{-1}\left(\boldsymbol{c}_{1}+\alpha \Sigma \boldsymbol{c}_{2}\right) \\
& \boldsymbol{u}_{2}=\left(I_{m}+\alpha^{2} \Sigma^{2}\right)^{-1}\left(\boldsymbol{c}_{2}-\alpha \Sigma \boldsymbol{c}_{1}\right) .
\end{aligned}
$$




\section{Some Remarks on the Computational Cost}

In this section we consider the computational cost of the algorithm described in the previous section for the solution of a single linear system and the cost to solve a different linear system changing just the vector $\boldsymbol{b}_{\alpha}$ and the parameter $\alpha$. We suppose that matrix $A$ has a dense structure, that is the worst case from the computational point of view. It is obvious that this process cannot be used when we have to solve a small number of linear systems since it involves the computation of the Schur decomposition of a matrix of order $n$ and of all the factor of the singular value decomposition of a bidiagonal matrix of order $n / 2$. It is well-known (see [6]) that the computational cost of the Schur decomposition of $A$ is

$$
2 n^{3}+3 n^{2}+7 n
$$

plus $n-1$ square roots, while the cost of the SVD decomposition of a bidiagonal matrix of order $m$ is

$$
12 m^{2}+30 m+2 m \text { square roots, }
$$

and, in this particular case is $3 n^{2}+15 n$ plus $n$ square roots. In the following we shall neglect the number of square roots. In Table 1 we recall the number of operation required by the decompositions we need to compute just once. In Table 2 we recall the computational costs required by the operations that we need to perform at each step (i.e. when the value of parameter $\alpha$ changes).

Table 1. Computational costs for the different steps of the algorithm

\begin{tabular}{lc}
\hline Step & Flops \\
\hline Schur Decomposition & $2 n^{3}+3 n^{2}+7 n$ \\
SVD Decomposition & $3 n^{2}+15 n$ \\
\hline Total & $2 n^{3}+6 n^{2}+22 n$ \\
\hline
\end{tabular}

We observe that the lower cost of the solution of the linear system is due to the special structure of the coefficient matrix. If we have to solve $K_{\alpha}$ linear systems (with $K_{\alpha}$ different values of $\alpha$ ) the global computational cost is

$$
N_{1}=2 n^{3}+6 n^{2}+22 n+K_{\alpha}\left(5 n^{2}+4 n\right)=2 n^{3}+\left(6+5 K_{\alpha}\right) n^{2}+2\left(11+2 K_{\alpha}\right) n .
$$

Now we compare the computational with a similar algorithm, which starts considering the same Schur decomposition of $A$ but solves, for a fixed value of $\alpha$ a tridiagonal non-symmetric system. In detail we consider the decomposition

$$
I+\alpha A=Q(I+\alpha H) Q^{\top}
$$


Table 2. Computational costs for each step the algorithm ( $\alpha$ fixed)

\begin{tabular}{lc}
\hline Step & Flops \\
\hline Step 1 (Matrix-vector product) & $2 n^{2}$ \\
Step 2 (Matrix-vector product) & $n^{2} / 2$ \\
Step 3 (Solution of the linear system) & $4 n$ \\
Step 2 (Matrix-vector products) & $5 n^{2} / 2$ \\
\hline Total & $5 n^{2}+4 n$ \\
\hline
\end{tabular}

hence the single linear system becomes

$$
Q(I+\alpha H) Q^{\top} \boldsymbol{x}_{\alpha}=\boldsymbol{b}_{\alpha}
$$

Once this decomposition has been computed the following steps need to be carried on:

1. Compute $\boldsymbol{y}_{\alpha}=Q^{\top} \boldsymbol{b}_{\alpha}$

2. Compute the $L U$ decomposition of the tridiagonal matrix $I+\alpha H$;

3. Solve the linear system $(I+\alpha H) \boldsymbol{u}_{\alpha}=\boldsymbol{y}_{\alpha}$;

4. Compute $\boldsymbol{x}_{\alpha}=Q \boldsymbol{u}_{\alpha}$.

Also for this algorithm we recall in Table 3 the computational costs for the single steps.

Table 3. Computational cost for the second algorithm

\begin{tabular}{lc}
\hline Step & Flops \\
\hline Schur decomposition of $A$ & $2 n^{3}+3 n^{2}+7 n$ \\
Step 1 (Matrix-vector product) & $2 n^{2}$ \\
Step 2-3 (LU decomposition + linear solver) & $11 n$ \\
Step 4 (Matrix-vector products) & $2 n^{2}$ \\
\hline
\end{tabular}

The total computational cost for $K_{\alpha}$ linear systems is

$$
N_{2}=2 n^{3}+3 n^{2}+7 n+K_{\alpha}\left(4 n^{2}+11 n\right)=2 n^{3}+\left(3+4 K_{\alpha}\right) n^{2}+\left(7+11 K_{\alpha}\right) n .
$$

Both costs have the same coefficient for $n^{3}$ hence it is important to analyze the coefficients for $n^{2}$ and $n$. Comparing (8) and (9) and in particulat their dependence on the number $K_{\alpha}$ of systems that must be solved we observe that the computational cost $N_{1}$ of the first algorithm, for large $K_{\alpha}$, will be greater than $N_{2}$. Just for small values of $n$, i.e. when the linear coefficient for $n, N_{1}$ is smaller than $N_{2}$. In order to underline this phenomenon in Figure 1 we have sketched the number of flops required by the two algorithms (solid line for the 


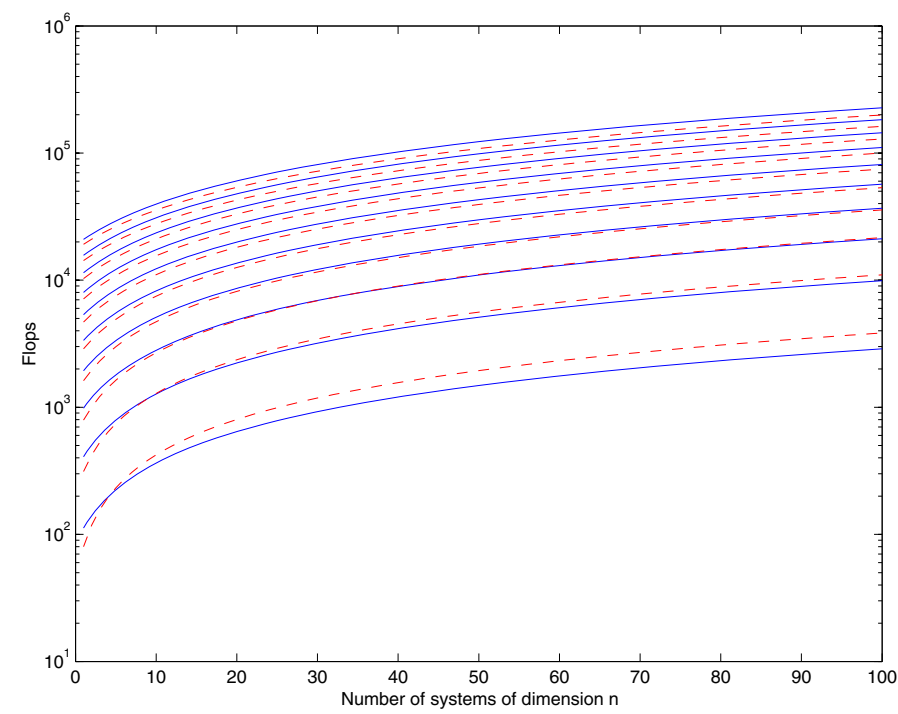

Fig. 1. Computational costs for considered algorithms

first, dashed line for the second), taking different value for $n=2,4, \ldots, 20$, and $K_{\alpha}$.

\section{Applications}

In this section we describe just an efficient application of the algorithm described in Section 2. Considering the Kortvieg-de Vrijes partial differential equation

$$
u_{t}=-u u_{x}-\delta^{2} u_{x x x},
$$

with periodic boundary conditions $u(0, t)=u(L, t)$, where $L$ is the period and $\delta$ is a small parameter. As shown in 4, appropriate methods of space discretization lead to solve a set of ODEs of the form

$$
y^{\prime}=A(y) y, \quad y(0)=y_{0},
$$

evolving on the sphere of radius $\left\|y_{0}\right\|$, where $y(t)=\left(u_{0}(t), u_{1}(t), \ldots, u_{N-1}(t)\right)^{\top}$, $u_{i}(t) \approx u(i \Delta x, t)$ for $i=0,1, \ldots, N-1$, and where $\Delta x=\frac{2}{N}$ is the spatial step of $[0,2]$. For instance if we consider the space discretization method in [8] we have

$$
A(y)=-\frac{1}{6 \Delta x} g(y)-\frac{\delta^{2}}{2 \Delta x^{3}} P
$$

where both $g(y)$ and $P$ are two $N \times N$ skew symmetric matrices given by:

$$
[g(y)]_{i, j}= \begin{cases}u_{i-1}+u_{i} & \text { if } j=i+1 \\ -\left(u_{0}+u_{N-1}\right) & \text { if } i=1, j=N \\ -\left(u_{j-1}+u_{j}\right) & \text { if } i=j+1 \\ u_{0}+u_{N-1} & \text { if } i=N, j=1 \\ 0 & \text { otherwise. }\end{cases}
$$


and

$$
P=\left[\begin{array}{cccccccc}
0 & -2 & 1 & 0 & \ldots & 0 & -1 & 2 \\
2 & 0 & -2 & 1 & \ddots & & 0 & -1 \\
-1 & 2 & 0 & -2 & \ddots & \ddots & & 0 \\
0 & -1 & 2 & 0 & \ddots & \ddots & \ddots & \vdots \\
\vdots & \ddots & \ddots & \ddots & \ddots & \ddots & 1 & 0 \\
0 & & \ddots & \ddots & 2 & 0 & -2 & 1 \\
1 & 0 & & \ddots & -1 & 2 & 0 & -2 \\
-2 & 1 & 0 & \ldots & 0 & -1 & 2 & 0
\end{array}\right]
$$

Applying the Backward Euler Method a system like (1) must be solved at each step, especially the paramter $\delta$ is time dependent and a splitting technique for (10) is used using an explicit method for $g(y)$ and an implicit one for $P$.

\section{References}

1. Bai Z., Golub G., Ng M.K.: Hermitian and skew-Hermitian splitting methods for non-Hermitian positive definite linear systems. SIAM J. Matr. Anal. 24 (3) (2003) 603-626

2. Benzi M., Bertaccini D.: Approximate inverse preconditioning for shifted linear systems. BIT 43 (2003) 231-244

3. Bertaccini D.: Efficient solvers for sequences of complex symmetric linear systems. ETNA 18 (2004) 49-64

4. Chen J.B., Munthe-Kaas H., Qin M.Z.: Square-conservative schemes for a class of evolution equations using Lie group methods. SIAM J. Num. Anal. 39 (6) (2002) 2164-2178

5. Del Buono N., Lopez L., Peluso R.: Computation of the exponential of large sparse skew-symmetric matrices. SIAM J. Sci. Comp. 27 (2005) 278-293

6. Golub G.H., Van Loan C.F.: Matrix Computation. The John Hopkins Univ. Press, Baltimore, (1996)

7. Saad Y.: Analysis of some Krylov subspace approximation to the matrix exponential operator, SIAM J. Numer. Anal. 29 (1) (1992) 209-228

8. Zabusky N.J., Kruskal M.D.: Interaction of solitons in a collisionless plasma and the recurrence of initial states. Phys. Rev. Lett. 15 (1965) 240-243 